\title{
ESTIMATION OF CROSS-FLOW INFLUENCE ON SPRING-MOUNTED CYLINDER IN TRIANGULAR CYLINDER ARRAY
}

\author{
Sabine Upnere ${ }^{1,2}$, Normunds Jekabsons ${ }^{2,3}$ \\ ${ }^{1}$ Riga Technical University, Latvia; ${ }^{2}$ Ventspils University College, Latvia; \\ ${ }^{3}$ Jekabsons Engineering Systems, Latvia \\ upnere@gmail.com,normunds@jesystems.eu
}

\begin{abstract}
Ongoing research is motivated by the need to better understand the cross-flow and cylinder mechanical interactions leading to vibration processes in close-packed triangular cylinder array. Computational Fluid Dynamics (CFD) simulations have been done with open source OpenFOAM software. Unsteady Reynolds Averaged Navier-Stokes equations for incompressible flows are solved by the finite volume method. Several two-equation turbulence models have been investigated: standard k-epsilon, RNG k-epsilon, and realisable kepsilon model. Based on the pressure drop comparison between the calculations and the experiment, the RNG kepsilon turbulence model has been chosen. CFD simulated quasi-steady-state flow serves as an initial condition for further transient modelling. A spring-mounted cylinder(s) is installed in an array of surrounding rigid cylinders. The neighbourhood rods' influence on the oscillating rod in the bundle system is studied numerically. Oscillations of the cylinder have been described with mass-spring motion equation. Flexible supports allow vibrating the cylinder in both, the longitudinal and transverse direction under the action of unsteady hydrodynamic forces. Dynamic changes of mesh cells ensure that cylinder motion acts on bypassing flow.
\end{abstract}

Keywords: cross-flow, cylinder array, flow-induced vibrations, OpenFOAM, turbulence.

\section{Introduction}

Due to their practical applications, flow-induced vibrations in tube array is the subject with a large number of investigations, however, the problem stays incompletely understood because of its high nonlinearity. Vibrations induced by bypassing flow usually are easily detectable and potentially can serve as an indicator of the structure integrity and wear.

Arrays of cylinders merged in liquid are widely used in engineering such as several offshore structures, heat exchangers, boilers etc. Heat exchangers are crucial in a number of agricultural fields, for example, in dairy products, food and beverages pasteurisation process, as well as in biofuel distillation. In some specific cases, cross flow induced vibrations can occur in the filtration process when high Reynolds fluid flows through the fibre system.

The cross flow of the fluid can couple with the rod array in such a way that the flow energy is transferred to the cylinders and excessive oscillations may develop. In the case of a circular cylinder arrays in water flow, the mechanisms of primary interest are turbulence buffeting and fluidelastic instability (FEI) [1]. Failure occurring at turbulence buffeting may take several years to develop, whereas FEI failure can induce in short-term, in extreme cases in a matter of hours. Vibrations can noticeably shorten a device lifespan due to fatigue or lead to the structure integrity problems.

Several simple analytical / semi-empirical FEI models for a single flexible cylinder in a fixed cylinder array are developed, for example, quasi-static by Connors [2], quasi-steady by Price and Paidoussis [3], unsteady theoretical model by Lever and Weaver [4] or model by Tanaka and Tahakara [5].

Due to increasing computer power, a useful approach for vibration prediction is Computational Fluid Dynamics (CFD) simulations. Two-dimensional CFD calculations of FEI in tube bundle with normal triangular arrangement have been done by Schröder and Gelbe [6]. Their calculations show that critical velocities for fluidelastic instability are lower than the experimental data and the RMSamplitudes are higher. Numerical prediction of FEI in normal triangular tube bundles with multiple flexible circular cylinders is described in [7]. One of the outputs is the conclusion that the upstream cylinders being flexible increase the instability of the downstream cylinder. However, the downstream cylinder impact on the upstream cylinder stability is less. The surface vorticity method (SVM) is applied to simulate the flow-induced vibrations of a single cylinder row and a staggered cylinder array in [8]. The SVM is suitable for large-amplitude vibrations at sub-critical Reynolds numbers. Forced oscillations and self-excited vibrations have been analysed by Pedro et al. [9]. The CFD calculations have numbers of advantages such as a) all interesting data (cylinder velocity and amplitude, flow velocity and pressure etc.) may be available at every location in the computational domain at any time; 
b) flexibility choosing the initial conditions such as the flow velocity, array configuration, flexible cylinder location etc. However, computations consume the immense computation time, even on highperformance computation clusters.

In general, the objective of this research is to better understand the cross-flow and the rod bundle mechanical interactions leading to vibration processes in close-packed triangular cylinder array using experimental and numerical methods. The obtained results will be used as input to develop reduced order models useful for the structure health monitoring. The paper describes the used CFD approach to predict the spring-mounted cylinder response to cross-flow induced forces. Three combinations of flexibly mounted cylinders are analysed: the case with one freely vibrating cylinder; the case with three vibrating cylinders and the case with five cylinders. The impact of neighbour cylinders on the oscillation amplitude of flexibly mounted cylinders has been investigated.

\section{Materials and methods}

Fluid flow is assumed to be incompressible and turbulent, thermal effects are neglected. The Reynolds number is $5.6 \cdot 10^{3}$. Open source CFD toolkit OpenFOAM 2.4.x has been utilised to solve two-dimensional differential equations of continuity and momentum. To get the equation system closure the (Unsteady) Reynolds Averaged Navier-Stokes ((U)RANS) approach is used. The following two-equation turbulence models are investigated: standard k-epsilon, RNG k-epsilon, and realisable k-epsilon model.

The motion of the cylinder can be modelled as a mass-spring-damper system (1). Build-in six degree-of-freedom OpenFOAM solver is used to simulate the motion of the rigid, flexibly-mounted cylinder. Rotation of the cylinder is not allowed; the cylinder can move only in an x-y plane.

$$
m \frac{d^{2} x_{i}}{d t^{2}}+c \frac{d x_{i}}{d t}+k x_{i}=F_{i}, i=1,2,
$$

where $m$ - mass of the oscillating body, including hydrodynamic added mass, $\mathrm{kg}$;

$c$ - damping coefficient, $\mathrm{N} \cdot \mathrm{s} \cdot \mathrm{m}^{-1}$

$k$ - stiffness coefficient, $\mathrm{N} \cdot \mathrm{m}^{-1}$;

$x$ - displacement, m;

$F-$ forces, N.

Constant Laplace's equation for the displacement is applied to recalculate the updated position of points through simulation. Mesh modifications are performed after each calculation step. A fluid dynamic force applied to moving the cylinder consists of the damping force due to fluid, stiffness force due to the rod displacement and the inertia force due to added fluid mass [10].

The experimental study of Weaver and El-Kashlan [11] in triangular tube bundle shows that the minimum number of tube rows required to investigate cross-flow induced phenomena typical of tube bank is six rows. In the present study, a cylinder array with seven rows is used. The total length of the computational domain is $0.416 \mathrm{~m}$. All cylinders have equal diameter of $d$. Part of the domain with the cylinder arrangement is shown in Fig. 1.

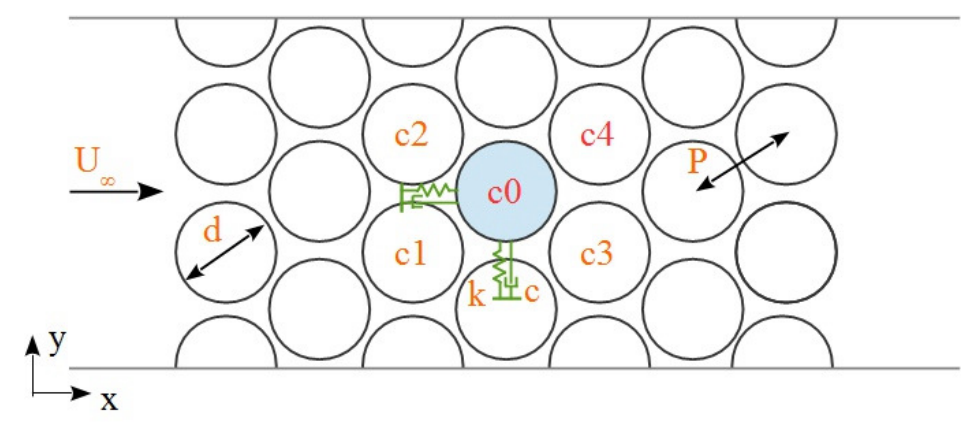

Fig. 1. Array configuration with central cylinder $\mathrm{c} 0$, upstream cylinders $\mathrm{c1}$, $\mathrm{c2}$ and downstream cylinders c3, c4: $U_{\infty}$ - freestream velocity; $d$ - cylinder diameter; $P$ - pitch length 
The spring-mounted circular cylinder is located in the middle of the fourth row based on the conclusion [11] that the critical tubes for fluidelastic instability are located in the third and fourth rows.

In order to model self-excited vibrations of the cylinder, each flexibly mounted cylinder has 2 springs, one for parallel and one for perpendicular movement (see an example of the cylinder $\mathrm{c} 0$ in Fig. 1). It can be freely moved in transverse and in-line direction due to fluid-dynamic forces. To contribute high amplitude oscillations, the damping coefficients, $c$ in both directions are set to zero. A cylinder with the natural frequency of $36 \mathrm{~Hz}$ and mass of $36.8 \mathrm{~g}$ is used as an oscillating body. Stiffness coefficients, $k$ for $\mathrm{c} 1, \mathrm{c} 2, \mathrm{c} 3$, and $\mathrm{c} 4$ are equal, for $\mathrm{c} 0$ the coefficient, $k$ is approximately three times smaller. Medium level of turbulence intensity, 5\%, is assumed. Water with density, $\rho=1000 \mathrm{~kg} \cdot \mathrm{m}^{-3}$ and kinematic viscosity $v=0.98 \cdot 10^{-6} \mathrm{~m}^{2} \cdot \mathrm{s}^{-1}$ is used as the working liquid media.

The applied boundary conditions are summarised in Table 1 . To decrease the computation time reduced domain was used and the effect of the channel walls was not taken into account using symmetry boundary conditions.

Table 1

Boundary conditions

\begin{tabular}{|c|l|}
\hline Boundary & \multicolumn{1}{|c|}{ Description } \\
\hline Inlet & Constant velocity specified by an equation, $U=U_{\infty}$ \\
\hline Outlet & $\begin{array}{l}\text { The outflow of fluid must be perpendicular to the } \\
\text { boundary, } p=p_{0}\end{array}$ \\
\hline Walls & Fluid has zero velocity relative to the boundary \\
\hline Oscillating surface & A tangential velocity is applied at no-slip walls \\
\hline Sides & Mirror symmetry \\
\hline
\end{tabular}

Due to the simulation complexity parallel calculation using High-Performance Computing cluster has been done.

\section{Results and discussion}

All simulations are done in two-dimensional space. A quasi-steady-state solution running simulations in a static cylinder array is obtained at first (see Fig. 2). The preliminary solution has been utilised to check the impact of the boundary conditions and mesh size and to choose the turbulence model. Experimental results of water channel measurements [12] are used for model validation. Comparison of the experimental and calculated pressure drop leads to the choice of the RNG k-epsilon turbulence model.

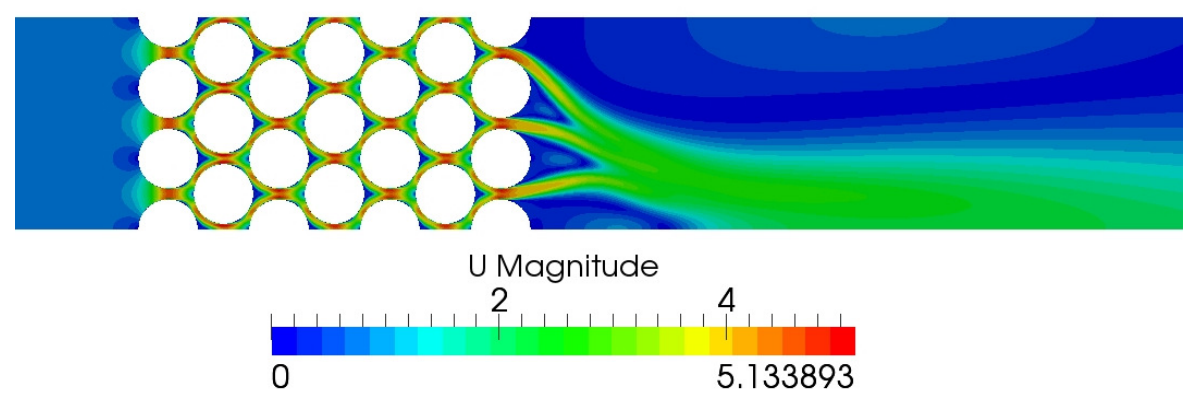

Fig. 2. Velocity field $\left(\mathrm{m}^{\cdot} \mathrm{s}^{-1}\right)$ distribution in the cylinder array

The results of the mesh study are summarised in Table 2, where $\Delta y$ is the height of the first cell near the wall. Mesh M3 is chosen for further simulations.

Mesh study

Table 2

\begin{tabular}{|c|c|c|}
\hline Mesh & $\boldsymbol{\Delta y}, \mathbf{m m}$ & Pressure drop \\
\hline M1 & 0.05 & $83.2 \%$ \\
\hline M2 & 0.02 & $97.9 \%$ \\
\hline M3 & 0.01 & $99.6 \%$ \\
\hline
\end{tabular}


Steady-state calculations have shown that the biggest fluid forces are on the first row cylinders (see Fig. 3). In the second row the hydrodynamic force is the smallest, only $\sim 39 \%$ of the first row. In the next two rows the forces increase to $\sim 46 \%$ and $\sim 49 \%$, respectively. In the 5th and 6th rows, the forces are between $\sim 51 \%$ and $\sim 52 \%$. In the last row the forces increase due to wake formation.

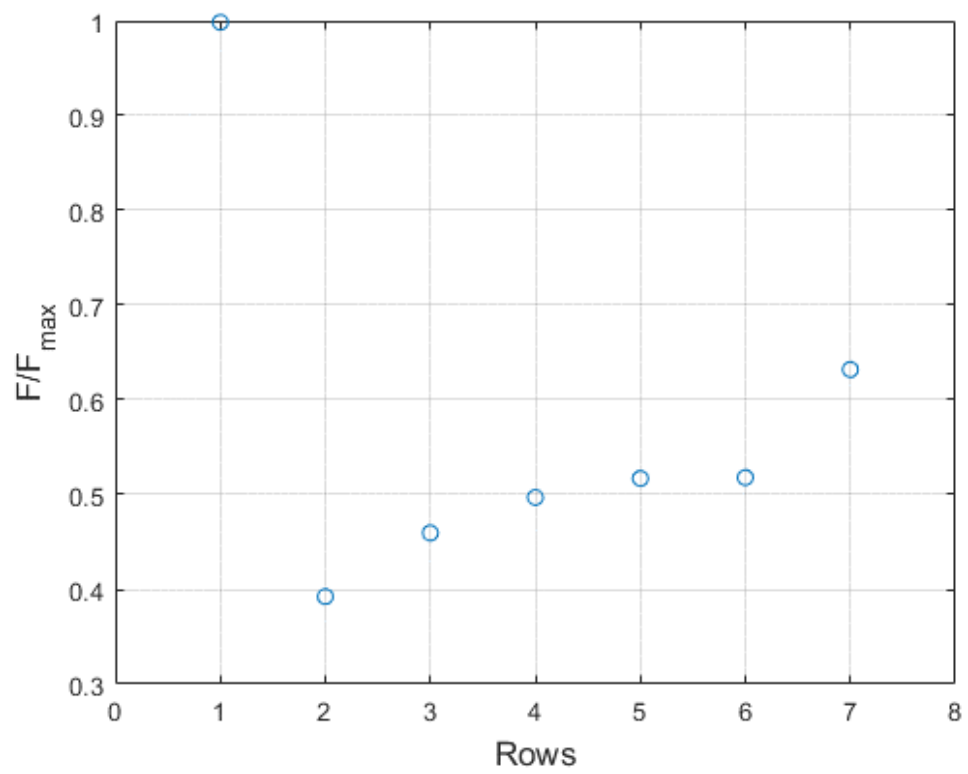

Fig. 3. Normalized averaged forces on cylinders depending on row

The pressure-velocity coupling is realised with the SIMPLE algorithm. Steady-state case convergence is achieved when residuals are at least $10^{-8}$. Converged solution is used as initial conditions for transient cases.

First order implicit time discretization scheme of Euler with maximum Courant number of 0.15 is applied for dynamic calculations. The maximum time step is $1 \cdot 10^{-4}$. The maximum cylinder oscillation frequency is $69.5 \mathrm{~Hz}$, therefore, there are $\sim 140$ steps per cycle. It can be considered to be small enough not to affect the simulation results based on [8] and [13]. In time-dependent cases for the pressurevelocity coupling the PIMPLE algorithm is utilised.

The following three cases with freely vibrating cylinders in the unsteady regime are analysed:

- Case I - only c0 can move in the x-y plane, other cylinders are static;

- Case II - c0 and two upstream cylinders, c1 and c2 being flexible;

- Case III - c0, two upstream, c1 and c2 and two downstream cylinders, c3 and c4 can freely vibrate.

The trajectories of the central cylinder, $\mathrm{c} 0$ depending on the neighbour cylinder dynamic conditions are presented in Fig. 4.

As seen from Fig. 4, the upstream and downstream cylinder motion increases the vibration amplitude of the central cylinder approximately two times. The major movement has been basically in the freestream flow direction. The calculation results show that the difference between Case II and Case III is relatively small regarding $\mathrm{c} 0$ motion amplitude. This is in line with [7] findings. Oscillation frequencies, $f_{\text {oscil }}$ of $\mathrm{c} 0$ in all cases are summarised in Table 3.

Table 3

\section{Oscillation frequencies of $\mathbf{c 0}$}

\begin{tabular}{|c|c|c|c|}
\hline Cases & Case I & Case II & Case III \\
\hline $\boldsymbol{f}_{\text {oscil }}$ & 69.63 & 83.87 & 80.25 \\
\hline
\end{tabular}

The frequency of the central cylinder increases from Case I to Case II, but decreases from Case II to Case III. The last one could be explained by the added mass effect. Further investigation is needed. 
In Case II and Case III, the cylinder $\mathrm{c} 0$ response is unstable. It is related to the upstream and downstream cylinders instability, see Fig. 5.

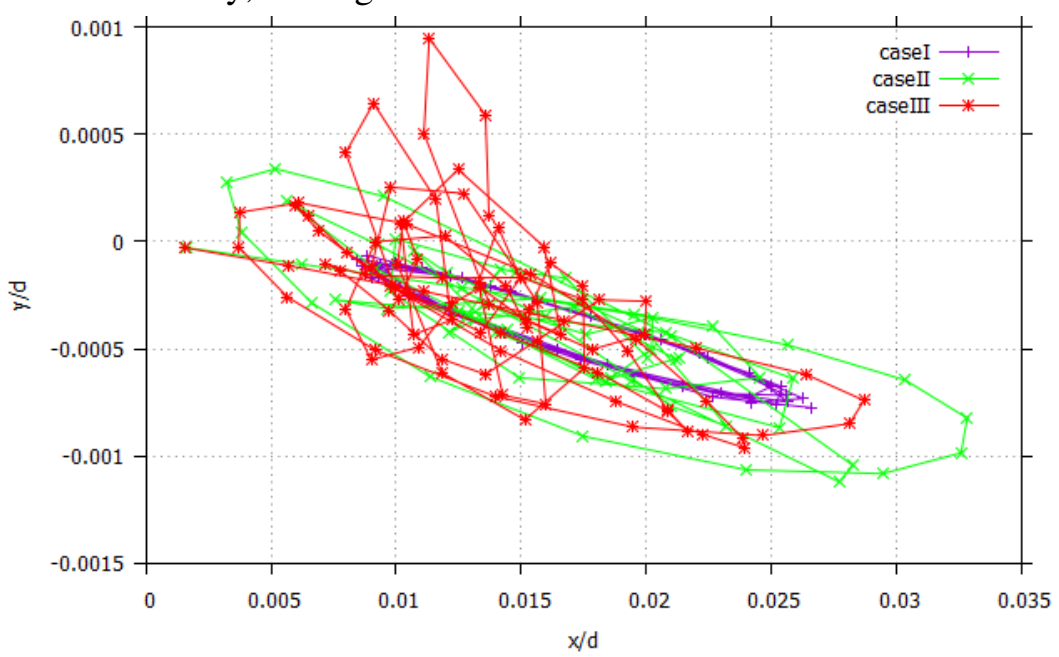

Fig. 4. Trajectories of $\mathbf{c 0}$ cylinders in $\mathbf{x}-\mathbf{y}$ plane: Case $\mathrm{I}-$ only $\mathrm{c} 0$ is spring-mounted; Case $\mathrm{II}-\mathrm{c} 0$, $\mathrm{c} 1$, and $\mathrm{c} 2$ are spring-mounted; Case III - c0, c1, c2, c3, c4 are spring-mounted

a)

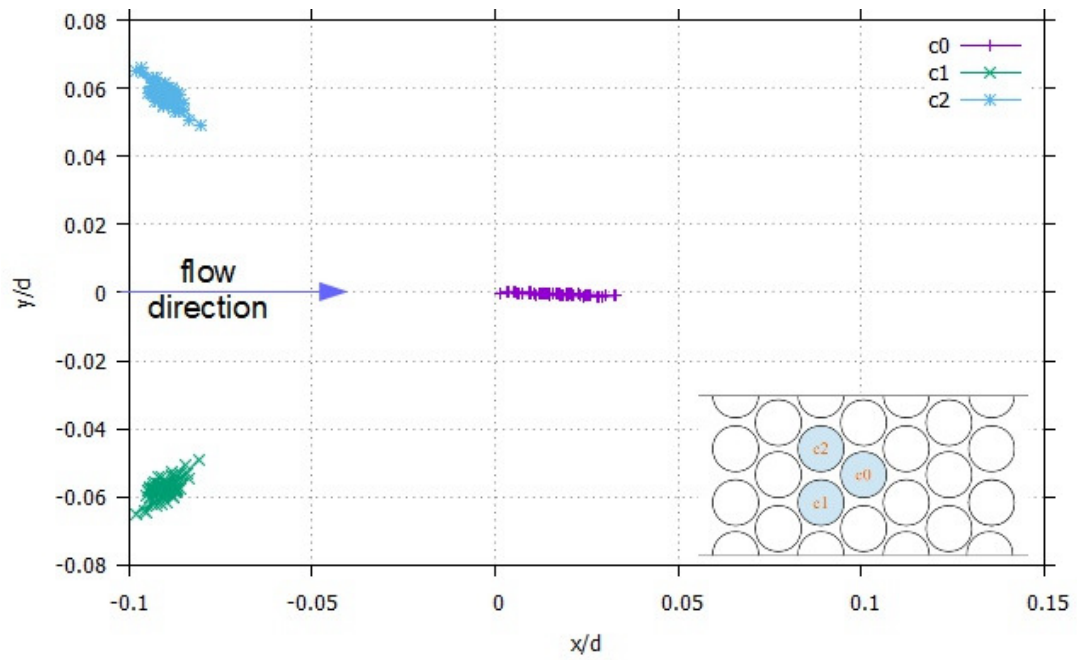

b)

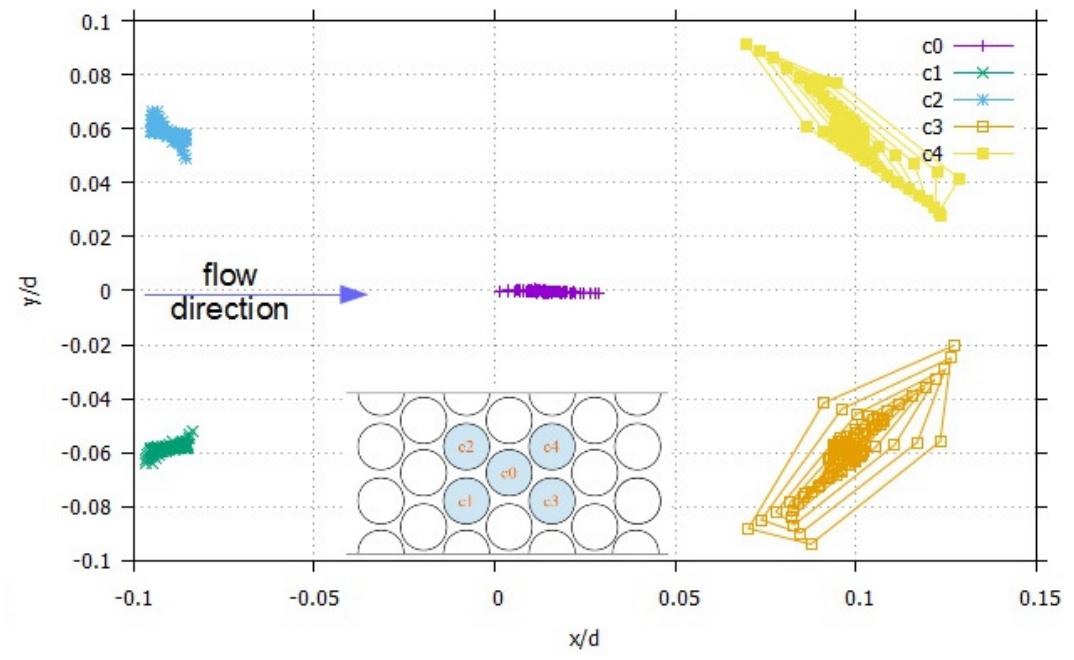

Fig. 5. Trajectories of oscillating cylinders in x-y plane: a - Case II with three oscillating cylinders; $\mathrm{b}$ - Case III with five oscillating cylinders 
In Case II the cylinders $\mathrm{c} 1$ and $\mathrm{c} 2$ move at approximately the same angle regarding the freestream flow direction, only in opposite way. If add two oscillating cylinders in downstream, the movement directions of $\mathrm{c} 1$ and $\mathrm{c} 2$ changes are minor comparing to Case II. The downstream cylinder motion amplitude is noticeably larger than the upstream one, although the stiffness coefficients and masses are equal. This could be explained by the increasing of the hydrodynamic forces in the downstream rows (see Fig. 3). The cylinders $\mathrm{c} 3$ and $\mathrm{c} 4$ move in opposite directions.

\section{Conclusions}

1. Two-dimensional Computational Fluid Dynamics calculations have been done to simulate selfexcited vibrations due to fluid forces in a closed-packed triangular cylinder array with a single and several flexibly mounted cylinders. A better understanding of the vibration formation mechanism could allow developing more powerful and safer systems. (U)RANS computations were performed using open source toolkit OpenFOAM solvers.

2. A quasi-steady solution has been obtained to find appropriate mesh refinement, turbulence model, boundary conditions etc. The calculations are validated with the experimental measurements. Static case results are used as initial conditions for time-dependent simulations.

3. In the cases of simulations with freely vibrating cylinders, the results show that the downstream cylinder impact on the upstream cylinder stability is smaller comparing to opposite situation. This is in line with other authors. The downstream cylinder motion amplitude is noticeably larger than the upstream at the same supporting conditions.

4. It should be noted that flow-induced vibrations are strongly dependent on the array configuration and other system properties and operating conditions.

\section{References}

1. Hassan M., Weaver D.S. Modeling of streamwise and transverse fluidelastic instability in tube arrays. Journal of Pressure Vessel Technology, vol. 138, 2016, pp 1-9.

2. Connors H. J. Fluidelastic vibration of tube arrays excited by cross flow. Proceedings of "ASME Winter Annual Meeting", 1970.

3. Paidoussis M.P., Price S.J., de Langre E. Fluid-structure interactions: cross-flow-induced instabilities. Cambridge, New York: Cambridge University Press, 2011, 402p.

4. Lever J.H., Weaver D.S. A theoretical model for fluid-elastic instability in heat exchanger tube bundles. Journal of Pressure Vessel Technology, vol. 104, 1982, pp 147-158.

5. Tanaka H., Takahara S. Fluid elastic vibration of tube array in cross flow. Journal of Sound and Vibration, vol. 77 (1), 1981, pp. 19-37.

6. Schröder K., Gelbe H. Two- and three-dimensional CFD-simulation of flow-induced vibration excitation in tube bundles. Chemical Engineering and Processing, vol. 38, 1999, pp. 621-629.

7. Jafari H.H., Dehkordi B.G. Numerical prediction of fluid-elastic instability in normal triangular tube bundles with multiple flexible circular cylinders. Journal of Fluids Engineering, vol. 135, 2013.

8. Lam K., Jiang G.D., Liu Y., So R.M.C. Simulation of cross-flow-induced vibration of cylinder arrays by surface vorticity method. Journal of Fluids and Structures, vol. 22, 2006, pp. 1113-1131.

9. de Pedro B., Parrondo J., Meskell C., Oro J.F. CFD modelling of the cross-flow through normal triangular tube arrays with one tube undergoing forced vibrations or fluidelastic instability. Journal of Fluids and Structures, vol. 64, 2016, pp. 67-86.

10. Kim S.N., Jung S.Y. Critical velocity of fluidelastic vibration in a nuclear fuel bundle. KSME International Journal, vol. 14 (8), 2000, pp. 816-822.

11. Weaver D.S., El-Kashlan M. On the number of tube rows required to study cross-flow induced vibrations in tube banks. Journal of Sound and Vibration, vol. 75 (2), 1981, pp. 265-273.

12. Upnere S., Jekabsons N., Dementjevs S. Analysis of cross-flow induced vibrations in staggered arrangement of multi-cylinder system. Proceedings of "5th European Seminar on Computing", June 5-10, 2016, Pilsen, Czech Republic, pp. 225.

13. Hassan M., Gerber A., Omar H. Numerical estimation of fluidelastic instability in tube arrays. Journal of Pressure Vessel Technology, vol. 132, 2010. 